\title{
DETERMINANTES INSTITUCIONAIS DO DESEMPENHO SOCIAL CORPORATIVO: COMPARAÇÃO ENTRE EMPRESAS NO BRASIL E CANADÁ
}

\author{
Rômulo Alves Soares \\ Doutorando em Administração e Controladoria \\ Universidade Federal do Ceará \\ Fortaleza - Ceará - Brasil \\ romuloalves61@gmail.com \\ Mônica Cavalcanti Sá de Abreu \\ Doutor em Engenharia de Produção \\ Universidade Federal do Ceará \\ Fortaleza - Ceará - Brasil \\ mabreu.ufc@gmail.com \\ Pedro de Barros Leal Pinheiro Marino \\ Mestre em Administração e Controladoria \\ Universidade de Brasília. \\ Brasília - Distrito Federal - Brasil \\ pedroblpmarino@gmail.com \\ Silvia Maria Dias Pedro Rebouças \\ Doutor em Estatística e Inv.Operacional -Prob. e Estatística \\ Universidade Federal do Ceará \\ Fortaleza - Ceará - Brasil \\ smdpedro@gmail.com
}

\section{RESUMO}

Esse estudo analisa a influência das características do ambiente institucional no qual as empresas se inserem no desempenho social corporativo. Para esse fim, realizou-se uma pesquisa descritiva e quantitativa, que utilizou dados secundários de empresas no Brasil e Canadá, que atuam nos setores de utilidade pública, petróleo e gás e materiais básicos. O desempenho social corporativo foi calculado a partir de informações dos relatórios de sustentabilidade publicados pelas empresas, as características financeiras foram retiradas dos relatórios anuais e as características institucionais foram medidas por meio de indicadores de diversos órgãos internacionais. Foram utilizadas técnicas de estatística descritiva, teste $t$ e regressão de dados em painel. Os resultados mostram que o controle de corrupção é determinante para influenciar o desempenho social corporativo das empresas no Brasil e Canadá, exercendo influência também sobre a sua dimensão ambiental. Para a dimensão exclusivamente social, observou-se um impacto significativo da qualidade da relação entre sindicatos e empregadores. $\mathrm{O}$ estudo mostra que forças isomórficas operam no campo institucional e afetam a adoção de um comportamento socialmente responsável. Situando o estudo em países institucionalmente distantes, tais como Brasil e Canadá, esse estudo reforça a influência do sistema nacional de negócios sobre o desempenho social corporativo.

Palavras-chave: Desempenho social corporativo. Sistema nacional de negócios. Teoria institucional.

\section{INSTITUTIONAL DETERMINANTS OF CORPORATE SOCIAL PERFORMANCE: COMPARISON BETWEEN COMPANIES IN BRAZIL AND CANADA}

\section{ABSTRACT}

This study analyses the influence of institutional environment on which companies act regarding their corporate social performance. In order to achieve this goal, a descriptive and quantitative research was conducted, based on data of Brazilian and Canadian companies, from one of those sectors: utility, materials, and oil and gas operations. Corporate social performance was measured using information disclosed on their sustainability reports, and their financial characteristics were accessed in their annual reports. Institutional environment characteristics were measured by indicators of several international organizations. Results showed that control of corruption is the main determinant of corporate social performance in Brazil and Canada, affecting mainly the environmental dimension. As for the social dimension, the quality of unionemployers relation showed a significant impact. This research emphasizes that managers must evaluate the institutional environment in which their companies are, while establishing their strategies. The study also shows that isomorphic forces operate in the institutional field and affect the adoption of socially responsible behavior. Placing the study in institutionally distant countries, such as Brazil and Canada, the study reinforces the influence of the national business system on corporate social performance.

Key words: Corporate social performance. Institutional theory. National business systems. 


\section{INTRODUÇÃO}

Decisões dos agentes econômicos são influenciadas por fatores ligados ao preço, como pressupõe o equilíbrio geral, mas também por fatores alheios ao preço, como as instituições (Amable, 2003). Huntington (1969) define instituições como padrões de comportamento estáveis, apreciados e recorrentes, que podem ser formais, como o governo ou as corporações, ou informais, como normas, incentivos e regras. Instituições criam, portanto, uma regularidade no comportamento humano e possibilitam o surgimento de padrões (Klein, 1998).

Neste sentido, as empresas têm, cada vez mais, dedicado recursos a várias iniciativas sociais, desde a sensibilização da comunidade, na qual se inserem, passando pela proteção ambiental, e por práticas de negócio socialmente responsáveis ( $\mathrm{Du}$, Bhattacharya \& Sen, 2010). Empresas identificam a possibilidade de obter vantagens estratégicas, por meio do investimento em responsabilidade social corporativa (RSC). Segundo Aguinis (2011), a RSC pode ser definida como um conjunto de ações e políticas, que levam em consideração as expectativas dos stakeholders (partes interessadas).

Estudiosos têm se voltado para o campo da RSC, e exploram uma possível relação entre o desempenho social corporativo (DSC) e o desempenho financeiro (Kim, Kim, \& Qian, 2018; Orlitzky, Schmidt, \& Rynes, 2003; Inoue \& Lee, 2011; Hasan, Kobeissi, Liu, \& Wang, 2018; Esteban-Sanchez, Cuesta-Gonzalez, \& Paredes-Gazquez, 2017). O desempenho social corporativo (DSC) reflete os resultados positivos oriundos do atendimento das expectativas dos stakeholders, e para tanto, os meios de produção devem ser empregados de forma a promover o bem-estar socioeconômico (Iannou \& Serafeim, 2012).

Aguinis e Glavas (2012) apontam algumas deficiências na literatura de DSC. Em geral, os estudos levam em consideração apenas um nível de análise. Ou seja, há a necessidade de pesquisas que sejam capazes de abordar a questão do desempenho social corporativo, de modo multidisciplinar, considerando de forma conjunta fatores organizacionais (características das empresas) e institucionais. Lattemann et al. (2009) reforçam que, a adoção de práticas socialmente responsáveis depende do ambiente institucional e do nível de desenvolvimento econômico dos países. Neste contexto, os sistemas nacionais de negócios (SNN) de países desenvolvidos e de países em desenvolvimentos ou emergentes resultam em diferentes expressões de RSC e, consequentemente, de DSC (Abreu et al., 2015; Jamali \& Neville, 2011; Jamali \& Karam, 2018).

Whitley (1999) aponta quatro elementos-chave que compõem os SNN: o sistema político; o sistema financeiro; os sistemas educativos e trabalhistas; e valores da cultura nacional. Karam e Jamali (2013) observam, com base nessa perspectiva, que há um distanciamento entre os SNN, de países desenvolvidos e em desenvolvimento, que contribui, de modo significativo, para a legitimidade de práticas de RSC. Nos países em desenvolvimento, o aspecto filantrópico é mais importante do que o legal e o ético, em decorrência de espaços vazios, como a ausência de instituições, normas e regulamentos necessários para o pleno funcionamento do mercado (Schrammel, 2013).

Campbell (2006) concorda com essa linha de pensamento, e afirma que boa parte dos estudos se preocupam em mostrar o impacto do gerenciamento dos stakeholders no desempenho social corporativo sem, no entanto, examinar os determinantes que influenciam a atuação socialmente responsável das corporações. Maignan e Ralston (2002) enfatizam a importância que os mecanismos institucionais exercem sobre a predisposição de uma empresa para adotar práticas de RSC. Neste sentido, Jamali e Mirshak (2007) alertam para a influência de diferentes contextos nacionais.

Diante do exposto, este estudo parte da seguinte pergunta de pesquisa: Características financeiras das empresas e fatores institucionais podem afetar o desempenho social corporativo? Para esse fim, foram levantados dados de 15 empresas brasileiras e 16 canadenses dos setores de materiais básicos, petroquímico e de utilidade pública, no período compreendido entre os anos de 2007 a 2014. Essa pesquisa justifica-se na necessidade de entender os principais fatores que afetam 
o DSC, especialmente, aqueles que estão fora do controle direto de seus gestores (Ioannou \& Serafeim, 2012). A escolha do Brasil e Canadá justifica-se pelo fato de serem países que diferem, de modo acentuado, quanto ao seu ambiente institucional.

Segundo dados do United Nations Development Programme (UNDP, 2016), o Brasil ocupa a $79^{\mathrm{a}}$ posição, com um IDH de 0,754 , enquanto, o Canadá é o $10^{\circ}$ melhor país em termos de desenvolvimento humano, com IDH de 0,92 . Quanto ao grau de liberdade econômica, a Heritage Foundation (2017) classifica o Brasil na $140^{\mathrm{a}}$ colocação entre 180 países, e coloca o Canadá como a sétima nação mais livre do mundo. Contudo, são semelhantes em termos econômicos. O Brasil é a $9^{\mathrm{a}}$ maior economia do mundo com PIB de US\$ 1,8 trilhão, enquanto, o Canadá ocupa a $10^{\mathrm{a}}$ posição com US\$ 1,6 trilhão, segundo dados do Banco Mundial.

Essas diferenças afetam significativamente vários aspectos das organizações dos países (Delmas, 2002), em especial as práticas sociais e ambientais adotadas. Para Thorne, Mahoney, Gregory e Convery (2017), as empresas canadenses se engajam em práticas sociais e ambientais voluntárias e alianças estratégicas em resposta às pressões dos stakeholders, para que hajam de modo responsável. Por outro lado, o Brasil, segundo Abreu, Cunha e Barlow (2015), apresenta um ambiente institucional no qual sociedade, empresas e governo não atuam de forma sistemática $\mathrm{e}$ integrada em direção à RSC.

Esse estudo traz duas contribuições. Primeiro, mostra como forças isomórficas, que operam no campo institucional, afetam a adoção de um comportamento socialmente responsável, e explora o efeito da heterogeneidade, entre os países, no isomorfismo local. E, contribui para o corpo limitado de conhecimentos sobre a influência do SNN no desempenho social corporativo, em países institucionalmente distantes, tais como Brasil e Canadá (Tschopp, Wells \& Barney, 2012; Orlitzk, Louche, Gond \& Chapple, 2017).

A próxima sessão apresenta as hipóteses sobre a influência das características financeiras, e dos sistemas político, financeiro, de educação e trabalho, no desempenho social corporativo. Em seguida, apresenta-se a metodologia adotada para realizar a modelagem econométrica em um painel de dados, no período de 2007 a 2014. Tomando por base dados coletados nos relatórios de empresas listadas na BM\&FBovespa (Brasil) e na Toronto Stock Exchange (Canadá), a amostra contém 227 observações, divididas em 110 de empresas no Brasil e 117 no Canadá. Finalmente, são apresentados e discutidos os resultados da pesquisa, os quais mostram diferentes pressões isomórficas no desempenho social corporativo, envolvendo a adoção de práticas ambientais e sociais.

\section{ENTENDENDO FATORES QUE INFLUENCIAM O DESEMPENHO SOCIAL CORPORATIVO}

O desempenho social corporativo (DSC) é uma resposta da organização com relação as expectativas e demandas dos stakeholders por práticas de responsabilidade social corporativa (Wood, 1991). O desempenho das organizações evoluiu de uma visão estreita, focada na maximização da riqueza do acionista (Friedman, 2000), para uma visão ampla, consistente com a teoria dos stakeholders (Freeman, 1984), que sugere que as organizações prestem contas a um público amplo, como funcionários, clientes, comunidades locais e governo, além de acionistas. Essa visão está de acordo com Surroca e Tribó (2008), os quais esclarecem que o conceito de DSC está relacionado ao objetivo de atender interesses de diferentes stakeholders. Uma abordagem de negócios para a DSC, considera vínculos estreitos e efetivos com as partes interessadas da empresa, aumentando sua reputação, o que pode melhorar sua posição no mercado e aumentar sua competitividade. Também pode abrir novos caminhos para o crescimento, permitindo que a empresa se expanda e obtenha lucros mais altos e melhor rentabilidade, ou seja, um melhor desempenho financeiro.

As práticas de RSC são modeladas pelas motivações dos gestores, shareholders e stakeholders, e a teoria institucional permite que essas práticas sejam estudadas e comparadas no 
seu contexto nacional, cultural e institucional (Matten $\&$ Moon, 2008). A teoria institucional atende aos aspectos mais resilientes da estrutura social, levando em consideração o processo pelo qual as estruturas, aqui incluídos os esquemas, regras, normas ou rotinas, se transformam em orientações impositivas do comportamento social (Scott, 2005). A abordagem dos sistemas nacionais de negócios (SNN) (Whitley, 1999) é uma das tradições preponderantes dentro da teoria institucional, e possibilita entender a adaptação das organizações aos ambientes institucionais, e as respostas em termos de padronização das formas organizacionais e práticas gerenciais.

Matten e Moon (2008) argumentam que o SNN é capaz de explicar o efeito das diferenças entre os países, com relação as práticas implícitas e explícitas de RSC. Práticas explícitas de RSC referem-se a práticas corporativas que assumem responsabilidade sobre algum interesse social. Normalmente, são constituídas de práticas voluntárias e estratégicas que combinam tanto ganho social quanto geração de valor para a empresa que a pratica. As práticas implícitas compreendem o papel desempenhado pelas empresas no contexto institucional no qual atuam, com o intuito de atender preocupações sociais. Constituem valores, normas e regras que se traduzem em obrigações das corporações com o bem-estar coletivo. É importante ressaltar que a diferença entre o que é RSC "explícito" e "implícito" reside também na intenção com a qual as empresas adotam determinada conduta corporativa.

\subsection{Influência das características financeiras no desempenho social corporativo}

A primeira característica da empresa capaz de influenciar o desempenho social corporativo é o tamanho. Empresas maiores atraem mais atenção do público, governo e stakeholders em geral, e, consequentemente, estarão sujeitas a maiores pressões para se comportarem de modo mais responsável (Chih, Chih, \& Chen, 2010; Lourenço \& Branco, 2013). Em geral, empresas maiores atuam em regiões demográficas e segmentos de mercado mais diversificados, o que aumenta a gama de stakeholders com os quais a empresa deve lidar. Empresas maiores também têm acesso a recursos com maior facilidade do que empresas menores, para se engajarem em atividades socialmente responsáveis (Brammer \& Pavelin, 2004).

De modo complementar, Wickert, Scherer e Spence (2016) identificam que, quanto maior for a empresa, maiores são os custos associados a práticas socialmente responsáveis. Práticas de RSC exigem uma capacidade de atendimento complexa, que só pode ser observada em grandes empresas. Em empresas menores, há ainda um agravante de serem mais propícias à interação entre propriedade e gestão, o que torna a adoção de práticas de RSC uma decisão discricionária. Por outro lado, para empresas maiores, cuja propriedade tende a não se confundir com a gestão, esta decisão tem um caráter mais instrumental. As afirmações sobre a relação entre o tamanho de uma empresa e seu desempenho social corporativo levam à formulação da primeira hipótese da pesquisa:

\section{Hipótese 1: O tamanho da empresa influencia positivamente o desempenho social corporativo.}

A segunda característica financeira capaz de influenciar o DSC é a rentabilidade. Ullmann (1985) argumenta que, em períodos de baixo desempenho econômico, ou seja, baixa rentabilidade, os gestores devem dar maior atenção aos objetivos financeiros da empresa, em detrimento de questões sociais. Por outro lado, elevados níveis de rentabilidade permitem que os gestores atendam as expectativas de seus stakeholders financeiros, e ainda disponham de recursos para investir em programas sociais.

Ruf et al. (2001) reforçam que empresas com melhor desempenho financeiro têm um maior custo de oportunidade. Simpson e Kohers (2002) levantam a hipótese de que os gestores, ao se depararem com um forte desempenho financeiro, preferem reduzir os investimentos socialmente responsáveis, a fim maximizar os lucros de curto prazo. Por outro lado, os gestores, ao se depararem com um desempenho financeiro abaixo do esperado, podem optar por investir em programas 
sociais, a fim de desviar a atenção sobre o aspecto financeiro. Com base no exposto, formula-se a segunda hipótese da pesquisa:

Hipótese 2: A rentabilidade da empresa influencia positivamente o desempenho social corporativo.

O endividamento é a terceira característica financeira que pode afetar o desempenho social corporativo. Segundo Artiach et al. (2010), o nível de débito na estrutura de capital de uma companhia revela uma medida do grau de importância de seus stakeholders financeiros. Fornecedores de crédito terão mais poder, conforme o número de empresa dependentes do financiamento por dívida (Roberts, 1992). À medida que o nível de endividamento da empresa aumenta, amplia-se a influência de seus credores (Artiach et al., 2010). Por outro lado, firmas com menores níveis de endividamento terão maior flexibilidade para financiar suas práticas sociais e ambientais (Ziegler \& Schröder, 2010; Lourenço \& Branco, 2013). Neste sentido, formula-se a terceira hipótese da pesquisa:

\section{Hipótese 3: O endividamento da empresa influencia negativamente o desempenho social corporativo.}

O desempenho social corporativo foi utilizado como variável dependente em 22 estudos dos 127 levantados por Margolis e Walsh (2003). Desses, 16 trabalhos encontraram uma relação positiva do desempenho social corporativo com o desempenho financeiro. Os autores criticam o fato de as pesquisas estarem mais voltadas para investigar como as práticas de RSC afetam o desempenho financeiro das empresas. Contudo, é necessário explorar a relação oposta, isto é, como as práticas de RSC são influenciadas, tanto pelo desempenho financeiro como por outras características da empresa.

\subsection{Influência do Sistema Nacional de Negócios no desempenho social corporativo}

\subsubsection{Influência do sistema político}

De acordo com Whitley (1999), uma das principais características institucionais na estruturação de um sistema nacional de negócios é a atuação do estado. O autor destaca que há diversas formas do Estado influenciar a formação de organizações econômicas. Destaca o fato de que, na convivência com o governo, empresas buscam barganhar contratos públicos, exercer pressão por meio de lobby e/ou pagar propinas. Por causa disso, Ioannou e Serafeim (2012) indicam que a eficiência do estado e sua burocracia, bem como os valores e crenças dos agentes públicos, afetam o desempenho social corporativo.

Luo (2006) indica que a corrupção em países emergentes é extremamente difundida, aumentando, de modo significativo, os custos de transação de empresas que desejam operar nesse mercado. Para o autor, atos ilícitos em ambientes corruptos são prejudiciais à governança corporativa e reputação das empresas, e põem em perigo eventuais práticas de RSC que poderiam ser adotadas, tendo forte repercussão legal, ética e organizacional.

Funderburk (2012) argumenta que a corrupção no Brasil é um problema sistêmico, que freia o desenvolvimento econômico e social do país de diversas formas. Para o autor, a corrupção existe em todas as formas possíveis no país: desde formas mais tradicionais como fraudes ordinárias, tráfico de influência, desvio de verba pública e pagamento de propinas; até formas mais sofisticadas como o financiamento ilegal de campanhas, mercados ilícitos e fraudes corporativas.

No Canadá, Klotz (2013) descreve a população do país como, em geral, seguidora da lei, porém desinformada. Justifica essa afirmação ao destacar que níveis de propina de agentes públicos dentro do país são quase inexistentes. No entanto, até 1999, o pagamento de propinas para agentes 
públicos de outros países não era considerado crime no Canadá, o que mudou com a publicação do Corruption of Foreign Public Officials Act (CFPOA). Desde então, o pagamento de propinas a agentes de outros países representa a principal forma de corrupção existente no país, mas com baixa ocorrências (Boisvert, Dent \& Quraishi, 2014). Entre 2010 e 2013, apenas 34 casos de corrupção dessa natureza foram descobertos e estão sendo investigados no país (Klotz, 2013).

Ioannou e Serafeim (2012) argumentam que em ambientes com elevados níveis de corrupção, as empresas têm maior propensão a adotar práticas antiéticas com o objetivo de reduzir seus custos, tais como empregar mão de obra ilegal, utilizar matéria-prima não regulamentada, ou aumentar sua participação no mercado, por meio de propina ou lobby. Esse comportamento pode estimular outras empresas a adotarem práticas antiéticas com o intuito de não perderem a competitividade. Com base no exposto, apresenta-se a quarta hipótese desta pesquisa:

\section{Hipótese 4: O controle da corrupção influencia positivamente o desempenho social corporativo.}

\subsubsection{Influência do sistema financeiro}

Além da corrupção, outro aspecto institucional crucial para a estruturação do sistema de negócios em um país é o sistema financeiro. Segundo Whitley (1999), o sistema financeiro influencia de diversas maneiras o SNN. A principal característica envolve o processo pelo qual o capital é disponibilizado e precificado, isto é, se a principal fonte de financiamento é o mercado de capitais ou de crédito. Um sistema baseado em mercados de capitais mobiliza e distribui capital por meio de um mercado, no qual a troca e o preço são ajustados de forma semelhante aos procedimentos de um mercado de commodities. Um sistema financeiro baseado em crédito apresenta instituições financeiras dominantes, que controlam as oportunidades de financiamento das empresas (Whitley, 1999).

Matten e Moon (2008) descrevem o sistema financeiro americano como sendo amplamente focado no mercado de capitais. A maior parte das grandes empresas tem esse mercado como sua fonte principal de financiamento, e a propriedade dessas empresas é bastante pulverizada. Nesse tipo de sistema, as empresas devem adotar um elevado nível de transparência e prestação de contas com seus investidores. No sistema europeu, as empresas estão envoltas por um número reduzido de grandes investidores, e entre eles, os bancos desempenham um papel importante.

O sistema financeiro brasileiro, segundo Brito, Corrar e Batistella (2007), é marcado pela dificuldade de acesso das empresas ao mercado de capitais. Studart (2003) explica que, desde a reforma bancária ocorrida no Brasil em 1988, o sistema financeiro do país passou a ser dominado por bancos. Com a estabilização da moeda ocorrida em 1994, houve uma rápida expansão na oferta de crédito, mas essa estabilização não mudou a preferência das instituições financeiras por financiamentos de curto prazo, sendo a oferta de crédito de longo prazo, em geral, restrita a bancos de desenvolvimento, como o Banco Nacional de Desenvolvimento (BNDES) (Prates \& Freitas, 2013). Brito, Corrar e Batistella (2007) comentam ainda que o sistema de crédito brasileiro é marcado por taxas de juros elevadas, limitando sua eficiência e dificultando o acesso de empresas ao crédito.

O sistema financeiro canadense, segundo Calmès (2004), é orientado ao mercado de capitais, com grande parte do financiamento das empresas realizado por meio de títulos de dívida e ações. Além disso, os intermediários financeiros têm se voltado para o mercado financeiro, na medida em que têm passado a oferecer serviços típicos desse mercado, como emissão de valores mobiliários.

De acordo com Ioannou e Serafeim (2012), em sistemas financeiros baseado num mercado de capitais bem desenvolvido, as empresas precisam garantir acordos financeiros mais vantajosos. Decisões nesse tipo de sistema tendem a ser orientadas por rentabilidade de curto prazo, e pode limitar práticas socialmente responsáveis, uma vez que ações desse tipo, podem levar um longo 
período para trazerem benefícios às empresas. Com base nessa argumentação, levanta-se a quinta hipótese desta pesquisa:

Hipótese 5: O grau com que um país adota um sistema financeiro baseado no mercado de capitais exerce uma influência positiva sobre o desempenho social corporativo.

\subsubsection{Influência do sistema educacional e trabalhista}

Além dos sistemas político e financeiro, Whitley (1999) também identifica como uma característica chave para a estruturação do sistema nacional de negócios, o sistema de desenvolvimento de habilidades e controle. Nesse sistema, destacam-se dois aspectos: o sistema educacional, que diz respeito ao desenvolvimento e certificação de competências e habilidades, e o segundo aspecto envolve o sistema de trabalho, que inclui as instituições que controlam como aqueles que detêm essas habilidades podem vendê-las no mercado de trabalho e definem como esse mercado é organizado.

Campbell (2007) afirma que as empresas estão mais predispostas a adotar práticas socialmente responsáveis se fizerem parte de sindicatos ou associações de empregados, desde que tais associações estejam orientadas para o desenvolvimento socialmente responsável. Além disso, as empresas também tendem a apresentar uma conduta socialmente responsável se houverem canais de comunicação institucionalizados entre empresas, sindicatos, grupos de empregados, comunidades, investidores e outros stakeholders. Com base nessa argumentação, levanta-se a sexta hipótese desta pesquisa:

Hipótese 6: Uma maior qualidade das relações entre empregados e empregadores influencia positivamente o desempenho social corporativo.

Concomitante ao sistema de trabalho, a capacidade de produção de capital humano qualificado também é uma característica importante para determinar a dimensão do desempenho social corporativo. Greening e Turban (2000) argumentam que investir em práticas socialmente responsáveis é uma forma de atrair mão de obra qualificada.

Ainda sob o aspecto educacional, Campbell (2007) destaca a importância da educação para o desempenho social corporativo. Empresas que atuam em ambientes onde haja uma maior pressão normativa, adotam condutas socialmente responsáveis. Essas pressões podem ser institucionalizadas, quando existir no país uma forte comunidade acadêmica capaz de cobrar esse tipo de conduta das empresas. Nessa mesma linha, Huang (2013) argumenta que o nível de educação dos gestores também é capaz de influenciar o desempenho social corporativo. Assim, a sétima hipótese para esta pesquisa é formulada:

Hipótese 7: O nível educacional de um país influencia positivamente o desempenho social corporativo.

De modo geral, entende-se, neste estudo, que determinadas características do ambiente institucional de um país influenciam as práticas de sustentabilidade de suas empresas (Abreu, et al., 2015; Chih, Chih \& Chen, 2010; Ioannou \& Serafeim, 2012). A análise desse ambiente, de modo comparativo entre Brasil e Canadá, contribui para o enriquecimento do estudo da RSC, ao demonstrar como os contextos institucionais afetam a dinâmica de práticas ligadas à RSC, o qual reflete no DSC.

\section{METODOLOGIA}

\subsection{Definição da amostra de empresas no Brasil e no Canadá}

Revista de Gestão Social e Ambiental - RGSA, São Paulo, v. 12, n. 3, p. 39-57, set./dez. 2018. 
Como critérios para a seleção da amostra foram selecionadas as empresas no Brasil e Canadá, que constavam na lista da Forbes 2000 do ano de 2008, resultando em 93 empresas, sendo 34 brasileiras e 59 canadenses. A lista da Forbes 2000 foi escolhida com o objetivo de selecionar empresas de porte semelhante em ambos os países, uma vez que tal característica pode influenciar no desempenho social corporativo. Desse grupo de empresas, foram selecionadas as que integram os setores de utilidade pública (utilities), materiais básicos (materials) e produção e distribuição de petróleo (oil \& gas operations). Dessa forma, a amostra utilizada na pesquisa é composta por 35 empresas, sendo 16 do Brasil e 19 do Canadá.

Além da escolha dos países, também se optou por limitar o número de setores analisados na pesquisa. Cai, Jo e Pan (2012) afirmam que o desempenho social corporativo pode variar consideravelmente entre setores, devido a diferenças na natureza do que é produzido, na legislação ambiental pertinente e mudanças nas normais sociais. Nesse sentido, alguns autores (Dos Reis \& Santos, 2015; Prno \& Slocombe, 2012) destacam a relevância dos setores de materiais básicos, energia elétrica e operações de petróleo e gás, devido ao seu elevado impacto ambiental, o que os sujeitam a regulamentações mais estritas, além de serem setores base da economia, servindo outras indústrias com insumos necessários ao seu funcionamento.

\subsection{Definição das variáveis dependentes e independentes}

A mensuração do desempenho social corporativo seguiu a metodologia proposta por Fischer e Sawczyn (2013), que tem como base a divulgação de indicadores de desempenho do GRI (2006). As variáveis dependentes do estudo são calculadas tendo como base pontuações das empresas nas dimensões ambiental (DA), social (DS), e para o desempenho social corporativo como um todo (DSC), sendo considerados na análise os percentuais relativos à pontuação máxima em cada dimensão. A dimensão ambiental do desempenho envolve 9 categorias e a dimensão social envolve 14. Em seguida, essas duas dimensões foram condensadas em uma dimensão total, a DSC. Estas dimensões, suas categorias e os indicadores GRI que as compõem são apresentadas nas Tabela 1.

Tabela 1 - Indicadores das dimensões ambientais e sociais utilizados na construção das variáveis dependente.

\begin{tabular}{|c|c|}
\hline Dimensão ambiental (pontuação máxima 54). & Indicador GRI \\
\hline Percentagem de materiais utilizados que são provenientes de reciclagem. & EN2 \\
\hline Consumo direto/indireto de energia, discriminado por fonte de energia primária. & EN3/EN4 \\
\hline Consumo total e descarte total de água, por fonte/destino. & EN8/EN21 \\
\hline $\begin{array}{l}\text { Descrição dos impactos significativos de atividades, produtos e serviços sobre a } \\
\text { biodiversidade das áreas protegidas e sobre as áreas de alto índice de } \\
\text { biodiversidade fora das áreas protegidas. }\end{array}$ & EN11/EN12 \\
\hline $\begin{array}{l}\text { Emissões totais diretas e indiretas de gases com efeito de estufa (Emissão de } \\
\text { substâncias destruidoras da camada de ozônio, NOx, SOx e outras emissões } \\
\text { atmosféricas significativas), por peso. }\end{array}$ & EN16/EN17/EN19/EN20 \\
\hline Quantidade total de resíduos, por tipo e método de eliminação. & EN22 \\
\hline $\begin{array}{l}\text { Iniciativas para mitigar os impactos ambientais de produtos e serviços e grau de } \\
\text { redução do impacto. }\end{array}$ & EN26 \\
\hline $\begin{array}{l}\text { Percentagem recuperada de produtos vendidos e respectivas embalagens por } \\
\text { categoria. }\end{array}$ & EN27 \\
\hline $\begin{array}{l}\text { Implicações financeiras e outros riscos e oportunidades para as atividades da } \\
\text { organização, devido às alterações climáticas. }\end{array}$ & $\mathrm{EC} 2$ \\
\hline Dimensão social (pontuação máxima 84). & Indicador GRI \\
\hline $\begin{array}{l}\text { Discriminação da mão de obra total, por tipo de emprego, por contrato de trabalho } \\
\text { e por região e taxa de rotatividade, por faixa etária, gênero e região. }\end{array}$ & LA1/LA2 \\
\hline $\begin{array}{l}\text { Taxa de lesões, doenças profissionais, dias perdidos, absenteísmo e óbitos } \\
\text { relacionados com o trabalho, por região. }\end{array}$ & LA7 \\
\hline $\begin{array}{l}\text { Média de horas por formação, por ano, por trabalhador, discriminadas por } \\
\text { categoria de funções. }\end{array}$ & LA10 \\
\hline Composição dos órgãos sociais da empresa e relação dos trabalhadores por & LA13 \\
\hline
\end{tabular}


categoria, de acordo com o gênero, a faixa etária, as minorias e outros indicadores de diversidade.

Razão entre o salário base de homens e mulheres, por categoria e funções.

Cobertura das obrigações referentes ao plano de benefícios definidos pela organização.

Número total de casos de discriminação e ações tomadas.

Casos em que exista um risco significativo de ocorrência de trabalho infantil, e medidas que contribuam para sua eliminação.

Casos em que exista um risco significativo de ocorrência de trabalho forçado ou escravo, e medidas que contribuam para sua eliminação.

Porcentagem e número total de unidades de negócio alvo de análise de risco à corrupção e medidas tomadas em resposta aos casos de corrupção.

Políticas, práticas e proporção de custos com fornecedores locais, em unidades operacionais importantes.

Desenvolvimento e impacto dos investimentos em infraestruturas e serviços que visam, essencialmente, o benefício público por meio de envolvimento comercial, em gêneros ou pro bono.

Estágios do ciclo de vida dos produtos e serviços em que os impactos de saúde e segurança são avaliados com o objetivo de efetuar melhorias, bem como a porcentagem das principais categorias de produtos e serviços sujeitas a tais procedimentos.

Tipo de informação sobre produtos e serviços exigida por regulamentos, e a percentagem de produtos e serviços significativos sujeitos a tais requisitos.

LA14

EC3

HR4

HR6

HR7

$\mathrm{SO} 2 / \mathrm{SO} 4$

EC6

EC8

PR1

PR3

\section{Dimensão total (pontuação máxima 138)}

Fonte: Fischer e Sawczyn (2013)

Cada categoria foi pontuada de 0 a 6 , seguindo a seguinte metodologia: 0 quando não é divulgada informação; 1 quando a informação de desempenho é apresentada em termos absolutos ou relativos. Soma-se mais 1 ponto quando, além da informação em termos absolutos ou relativos, ela também é dada: em relação a empresas semelhantes/rivais ou setor; em comparação com períodos passados; em relação às metas estipuladas; de forma normalizada ou de forma desagregada. Para as categorias que apresentam mais de um indicador (ex.: Categoria 2 formada pelo indicador EN3 e EN4), cada um foi avaliado separadamente e depois foi considerada a maior pontuação entre eles. O resultado obtido para a dimensão total da evidenciação foi dividido pela pontuação máxima, resultando em um indicador com valor entre 0 e 1 .

Para as variáveis independentes, foram escolhidos indicadores capazes de representar cada um dos aspectos chaves do ambiente institucional, as quais foram apresentados no referencial teórico. A tabela 2 mostra os indicadores selecionados para a características financeiras, seu efeito esperado com base nas hipóteses levantadas, bem como a fonte de coleta dos dados. Na tabela 3 são apresentadas as variáveis escolhidas para cada um dos SNN.

Tabela 2 - Variáveis independentes das características financeiras

\begin{tabular}{|c|c|c|c|c|}
\hline Variável & Efeito & Sigla & Operacionalização & Fonte dos dados \\
\hline Tamanho $\left(\mathrm{H}_{1}\right)$ & + & TAM & $\ln ($ Ativo Total $)$ & Relatório Anual \\
\hline $\begin{array}{c}\text { Rentabilidade } \\
\left(\mathrm{H}_{2}\right)\end{array}$ & + & RENT & $\frac{\text { LAIR }}{\text { Ativo Total }}$ & Relatório Anual \\
\cline { 1 - 2 } $\begin{array}{c}\text { Endividamento } \\
\left(\mathrm{H}_{3}\right)\end{array}$ & - & ENDIV & $\frac{\text { Passivo Circulante }+ \text { Passivo Não Circulante }}{2}$ Relatório Anual \\
\hline
\end{tabular}

Fonte: Elaborada pelos autores

Tabela 3 - Variáveis independentes dos fatores institucionais

\begin{tabular}{|c|c|c|c|c|}
\hline Variável & Efeito & Sigla & Operacionalização & Fonte dos dados \\
\hline Educação $\left(\mathrm{H}_{3}\right)$ & + & EDUC & $\begin{array}{c}\text { Média de anos de estudo da } \\
\text { população acima de } 25 \text { anos }\end{array}$ & $\begin{array}{c}\text { Programa das Nações } \\
\text { Unidas para o } \\
\text { Desenvolvimento }\end{array}$ \\
\hline Controle de corrupção $\left(\mathrm{H}_{4}\right)$ & + & CCORRUP & Corruption Perception Index & $\begin{array}{c}\text { Transparency } \\
\text { International }\end{array}$ \\
\hline
\end{tabular}

Revista de Gestão Social e Ambiental - RGSA, São Paulo, v. 12, n. 3, p. 39-57, set./dez. 2018. 


\begin{tabular}{|c|c|c|c|c|}
\hline $\begin{array}{c}\text { Financiamento pelo } \\
\text { mercado financeiro }\left(\mathrm{H}_{5}\right)\end{array}$ & + & MERC & $\begin{array}{c}\text { Valor de mercado das } \\
\text { empresas negociadas em bolsa } \\
\text { sobre o PIB }\end{array}$ & Banco Mundial \\
\hline $\begin{array}{c}\text { Qualidade das relações } \\
\text { sindicato-empregador }\left(\mathrm{H}_{6}\right)\end{array}$ & + & TRAB & $\begin{array}{c}\text { Índice de qualidade das } \\
\text { relações entre sindicatos e } \\
\text { empregadores }\end{array}$ & $\begin{array}{c}\text { World Economic } \\
\text { Forum }\end{array}$ \\
\hline
\end{tabular}

Fonte: Elaborada pelos autores

\subsection{Estimação dos modelos econométricos e análise dos dados}

A partir das variáveis elencadas, foram estabelecidos modelos a fim de testar as hipóteses levantadas nesta pesquisa. Todos os modelos aqui definidos atendem a todas as hipóteses do estudo, e cada um apresenta um desdobramento identificado por "A", para a dimensão ambiental, e "S", para dimensão social. Também cabe ressaltar que, em cada etapa, é feita a análise de regressão apenas com as variáveis independentes que medem as características financeiras, e, em seguida, com todas as variáveis do estudo.

Nesta pesquisa, tendo sido coletadas informações para os mesmos indivíduos ao longo de vários anos, optou-se pelo modelo de regressão de dados em painel. A decisão entre os painéis de efeitos fixos, aleatórios ou pooled OLS, se deu por meio dos testes de Hausman, Breush-Pagan e teste F para efeitos individuais. Em cada modelo, é utilizado o tipo de efeito mais adequado.

$$
\begin{gathered}
D S C_{i t p}=\beta_{0 i}+\beta_{1} T_{A M M_{i t p}}+\beta_{2} R E N T_{i t p}+\beta_{3} E N D I V_{i t p}+\varepsilon_{i t p} \\
D S C_{i t p}=\beta_{0 i}+\beta_{1} T A M_{i t p}+\beta_{2} R E N T_{i t p}+\beta_{3} E N D I V_{i t p}+\beta_{4} L I B E R_{t p}+\beta_{5} E D U C_{t p} \\
+\beta_{6} \text { CORRUP }_{t p}+\beta_{7} T R A B_{t p}+\beta_{8} M E R C_{t p}+\varepsilon_{i t p}
\end{gathered}
$$

Antes da análise de regressão, foi feita uma análise descritiva dos dados, na qual foram extraídas medidas de tendência e variabilidade, para que se pudesse ter uma ideia prévia de como os dados se comportam. Além disso, também foi empregado o teste $\mathrm{T}$ de diferença de médias, para comparação das empresas brasileiras e canadenses quanto ao seu perfil de desempenho social e ambiental. Optou-se pelo teste $\mathrm{T}$, uma vez que foi constatada a normalidade das variáveis empregadas no estudo, nos dois países, por meio do teste de Shapiro-Wilk.

Todas as análises foram executadas pelo software R (R Core Team, 2017). As análises descritivas e inferenciais foram executadas pelo pacote stats (R Core Team, 2017). As regressões de dados em painel e seus respectivos testes foram feitos com o pacote $\mathrm{plm}$ (Croissant \& Millo, 2008).

\section{RESULTADOS}

A Tabela 4 traz a quantidade de observações segregadas por país, setor e ano. Percebe-se que há um equilíbrio ao longo do período de análise para a amostra como um todo, em que o número de observações variou entre 25 e 32 . No caso do Brasil, o número de observações variou entre 13 e 15. Para o Canadá, houve uma maior discrepância, posto que o número de empresas oscilou entre 11 e 18. Na distribuição entre países, o Canadá apresentou maior número de empresas divulgando seus relatórios entre os anos de 2009 e 2013, sendo superado pelo Brasil em 2007 e 2008, havendo um empate nos anos de 2010 e 2014. Destaca-se, ainda, que no Canadá, no ano de 2011, todas as empresas estudadas divulgaram relatório de sustentabilidade. 
Tabela 4 - Número de observações por país, setor e ano.

\begin{tabular}{c|c|ccccccccc}
\hline País & Setor & 2007 & 2008 & 2009 & 2010 & 2011 & 2012 & 2013 & 2014 \\
\hline \multirow{5}{*}{ Brasil } & Materiais básicos & 6 & 6 & 6 & 6 & 5 & 4 & 4 & 6 \\
& Petróleo e gás & 2 & 2 & 2 & 2 & 2 & 2 & 2 & 2 \\
& Utilidade pública & 5 & 6 & 5 & 7 & 7 & 7 & 7 & 7 \\
\cline { 2 - 11 } & Total & 13 & 14 & 13 & 15 & 14 & 13 & 13 & 15 \\
\hline \multirow{5}{*}{ Canadá } & Materiais básicos & 6 & 5 & 6 & 6 & 9 & 9 & 8 & 8 \\
& Petróleo e gás & 5 & 5 & 7 & 7 & 7 & 6 & 5 & 5 \\
& Utilidade pública & 1 & 1 & 1 & 2 & 2 & 2 & 2 & 2 \\
\cline { 2 - 10 } & Total & 12 & 11 & 14 & 15 & 18 & 17 & 15 & 15 \\
\hline
\end{tabular}

Fonte: Elaborada com base nos dados da pesquisa

Quanto às características do ambiente institucional dos países, na Tabela 5 é apresentada a evolução histórica de cada uma delas. Destaca-se que o Canadá apresenta sempre valores superiores aos do Brasil. Destaca-se ainda o fato de que, no caso brasileiro, o indicador ligado ao mercado financeiro apresenta uma tendência decrescente desde 2009, e o indicador de trabalho, desde 2011.

No Canadá, apenas o controle de corrupção apresenta um período mais acentuado de decréscimo, que se observa desde 2010. Fica evidente a diferença entre os dois países que agrupam as empresas da amostra. De um lado, há um país com instituições mais fortes e bem definidas, o Canadá, e de outro, um país com elementos institucionais mais frágeis alguns, inclusive, se deteriorando ao longo dos anos.

Tabela 5 - Evolução temporal das características institucionais (variáveis independentes)

\begin{tabular}{c|c|cccccccc}
\hline Variável & País & 2007 & 2008 & 2009 & 2010 & 2011 & 2012 & 2013 & 2014 \\
\hline \multirow{2}{*}{ Ccorrup } & Brasil & 3,5 & 3,5 & 3,7 & 3,7 & 3,8 & 4,3 & 4,2 & 4,3 \\
& Canadá & 8,7 & 8,7 & 8,7 & 8,9 & 8,7 & 8,4 & 8,1 & 8,1 \\
\hline \multirow{2}{*}{ Merc } & Brasil & 98,12 & 34,93 & 80,34 & 69,95 & 46,99 & 50,87 & 42,66 & 35,97 \\
& Canadá & 149,98 & 66,99 & 122,32 & 134,47 & 106,89 & 112,34 & 114,95 & 117,27 \\
\hline \multirow{2}{*}{ Educ } & Brasil & 6,8 & 6,9 & 7,1 & 7,2 & 7,2 & 7,2 & 7,2 & 7,7 \\
& Canadá & 12,3 & 12,3 & 12,3 & 12,3 & 12,3 & 12,3 & 12,3 & 13 \\
\hline \multirow{2}{*}{ Trab } & Brasil & 4,3 & 4,2 & 4,1 & 4,2 & 4,3 & 4,1 & 3,7 & 3,6 \\
& Canadá & 4,9 & 4,9 & 5 & 5,1 & 5 & 4,9 & 5 & 5,1 \\
\hline
\end{tabular}

Fonte: Elaborada com base nos dados da pesquisa

Depois de estudada a composição da amostra, e visto que há um certo equilíbrio da distribuição das observações ao longo dos anos e entre os países, as estatísticas descritivas foram calculadas para cada país separadamente, e seus valores médios foram comparados por meio do teste t. Os resultados do DSC são apresentados na Tabela 6. Em geral, as medidas de tendência central, média e mediana, são maiores para as empresas brasileiras, quando comparadas às empresas canadenses. Apesar disso, a diferença observada não é estatisticamente significante, seja para o período completo, ou para cada um dos anos separadamente.

\section{Tabela 6 - Comparação da variável DSC entre Brasil e Canadá}

\begin{tabular}{c|ccccc|ccccc|c}
\hline \multirow{2}{*}{ Ano } & \multicolumn{9}{|c|}{ Empresas no Brasil } & \multicolumn{7}{c|}{ Empresas no Canadá } & \multirow{2}{*}{ Teste t } \\
\cline { 2 - 10 } & Média & Desvio & Mínimo & Mediana & Máximo & Média & Desvio & Mínimo & Mediana & Máximo & \\
\hline 2007 & 0,2004 & 0,1218 & 0,0161 & 0,2043 & 0,4194 & 0,1872 & 0,0859 & 0,0538 & 0,1882 & 0,3441 & $-0,2331$ \\
2008 & 0,1893 & 0,1211 & 0,0161 & 0,1774 & 0,4140 & 0,1828 & 0,0891 & 0,0538 & 0,1505 & 0,3495 & 0,2857 \\
2009 & 0,2058 & 0,1187 & 0,0108 & 0,2097 & 0,4140 & 0,1903 & 0,0944 & 0,0215 & 0,1935 & 0,3656 & $-0,1030$ \\
2010 & 0,1728 & 0,1049 & 0,0108 & 0,1828 & 0,3172 & 0,1548 & 0,0648 & 0,0323 & 0,1586 & 0,2419 & 1,2052 \\
2011 & 0,1530 & 0,0971 & 0,0323 & 0,1398 & 0,2742 & 0,2068 & 0,1203 & 0,0591 & 0,1935 & 0,4194 & 0,0107 \\
2012 & 0,1974 & 0,1017 & 0,0323 & 0,2151 & 0,3548 & 0,1759 & 0,0677 & 0,0645 & 0,1801 & 0,3172 & 0,3945 \\
2013 & 0,2070 & 0,1066 & 0,0376 & 0,2070 & 0,3441 & 0,1998 & 0,0956 & 0,0699 & 0,1828 & 0,4194 & $-0,2914$ \\
2014 & 0,2247 & 0,1147 & 0,0376 & 0,2419 & 0,4032 & 0,2118 & 0,0886 & 0,0806 & 0,2043 & 0,3925 & $-0,1330$ \\
\hline
\end{tabular}

Fonte: Elaborada com base nos dados da pesquisa 
Na Tabela 7, a dimensão social do DSC é comparada, e suas estatísticas descritivas segregadas por país. Nesta dimensão, as empresas brasileiras apresentaram valores maiores. Apesar de as diferenças serem maiores do que as observadas no desempenho social corporativo, apenas a diferença observada no ano de 2010 foi estatisticamente significante a $10 \%$. Dando continuidade, a Tabela 8 apresenta a comparação da dimensão ambiental do DSC, segregando as empresas brasileiras e canadenses. Diferente do desempenho social corporativo como um todo e de sua dimensão social, a dimensão ambiental apresentou médias maiores, bem como medianas, para as empresas canadenses, em comparação às brasileiras. Apesar disso, assim como no DSC, nenhuma diferença observada ao longo dos anos, ou mesmo para o período completo, pode ser considerada estatisticamente significante.

Após as análises descritiva e inferencial, averiguou-se, por meio da regressão de dados em painel, como as variáveis independentes e de controle podem influenciar o DSC e suas dimensões. Os modelos especificados na metodologia foram aplicados às empresas de cada país separadamente, bem como na amostra completa. Os resultados são mostrados nas tabelas 9 (DSC), 10 (DS) e 11 (DA).

Tabela 7 - Comparação da dimensão social entre empresas no Brasil e Canadá

\begin{tabular}{c|ccccc|ccccc|c}
\hline \multirow{2}{*}{ Ano } & \multicolumn{5}{|c|}{ Empresas no Brasil } & \multicolumn{5}{|c|}{ Empresas no Canadá } & \multirow{2}{*}{ Teste t } \\
\cline { 2 - 10 } & Média & Desvio & Mínimo & Mediana & Máximo & Média & Desvio & Mínimo & Mediana & Máximo & \\
\hline 2007 & 0,2083 & 0,1221 & 0,0208 & 0,1979 & 0,4063 & 0,1471 & 0,0843 & 0,0000 & 0,1354 & 0,3229 & 0,5577 \\
2008 & 0,1890 & 0,1131 & 0,0208 & 0,1927 & 0,4063 & 0,1549 & 0,0808 & 0,0417 & 0,1667 & 0,3229 & 0,9884 \\
2009 & 0,1935 & 0,1252 & 0,0104 & 0,2135 & 0,4271 & 0,1458 & 0,0891 & 0,0208 & 0,1458 & 0,3333 & 0,7067 \\
2010 & 0,1719 & 0,1081 & 0,0104 & 0,2031 & 0,3333 & 0,1183 & 0,0640 & 0,0104 & 0,0990 & 0,2292 & $1,9028^{*}$ \\
2011 & 0,1392 & 0,1150 & 0,0104 & 0,0938 & 0,2917 & 0,1722 & 0,1136 & 0,0417 & 0,1458 & 0,3646 & 1,0364 \\
2012 & 0,1808 & 0,1219 & 0,0000 & 0,2031 & 0,3750 & 0,1496 & 0,0717 & 0,0000 & 0,1458 & 0,2708 & 1,2411 \\
2013 & 0,1988 & 0,1081 & 0,0208 & 0,2031 & 0,3229 & 0,1587 & 0,0927 & 0,0313 & 0,1250 & 0,3646 & 0,0651 \\
2014 & 0,2076 & 0,1120 & 0,0208 & 0,2135 & 0,3854 & 0,1799 & 0,0859 & 0,0208 & 0,1875 & 0,3542 & 0,5015 \\
\hline
\end{tabular}

Fonte: Elaborada com base nos dados da pesquisa.

Níveis de significância: $*<0,1 ; * *<0,05 ; * * *<0,01$

Tabela 8 - Comparação da dimensão ambiental entre empresas no Brasil e Canadá

\begin{tabular}{c|ccccc|ccccc|c}
\hline \multirow{2}{*}{ Ano } & \multicolumn{5}{|c|}{ Empresas no Brasil } & \multicolumn{5}{|c|}{ Empresas no Canadá } & \multirow{2}{*}{ Teste T } \\
\cline { 2 - 10 } & Média & Desvio & Mínimo & Mediana & Máximo & Média & Desvio & Mínimo & Mediana & Máximo & \\
\hline 2007 & 0,1919 & 0,1280 & 0,0111 & 0,1889 & 0,4333 & 0,2299 & 0,0999 & 0,0778 & 0,2278 & 0,3889 & $-0,9613$ \\
2008 & 0,1897 & 0,1367 & 0,0111 & 0,1722 & 0,4222 & 0,2126 & 0,1133 & 0,0667 & 0,1889 & 0,3889 & $-0,3981$ \\
2009 & 0,2190 & 0,1231 & 0,0111 & 0,2444 & 0,4000 & 0,2378 & 0,1093 & 0,0222 & 0,2222 & 0,4000 & $-0,8943$ \\
2010 & 0,1738 & 0,1086 & 0,0111 & 0,2056 & 0,3222 & 0,1937 & 0,0751 & 0,0556 & 0,2056 & 0,2778 & 0,4565 \\
2011 & 0,1677 & 0,0905 & 0,0556 & 0,1889 & 0,3111 & 0,2437 & 0,1344 & 0,0444 & 0,2333 & 0,4778 & $-1,0391$ \\
2012 & 0,2151 & 0,1035 & 0,0556 & 0,2500 & 0,3444 & 0,2040 & 0,0890 & 0,0778 & 0,2111 & 0,3667 & $-0,4736$ \\
2013 & 0,2157 & 0,1182 & 0,0556 & 0,2222 & 0,3778 & 0,2436 & 0,1036 & 0,0889 & 0,2444 & 0,4778 & $-0,6254$ \\
2014 & 0,2429 & 0,1228 & 0,0556 & 0,2556 & 0,4222 & 0,2459 & 0,1003 & 0,1222 & 0,2333 & 0,4333 & $-0,7316$ \\
\hline
\end{tabular}

Fonte: Elaborada com base nos dados da pesquisa

$\mathrm{Na}$ Tabela 9, ao se analisar os modelos de regressão em que se consideram apenas as variáveis financeiras, constata-se que o endividamento se mostra significante (a 1\%) e negativo para o Brasil. A variável tamanho mostra-se significante (a 5\%) para a amostra canadense e para todas as empresas em conjunto, em ambos os casos de modo positivo. 
Tabela 9 - Regressão de dados em painel para o desempenho social corporativo (DSC)

\begin{tabular}{c|cc|cc|cc}
\hline \multirow{2}{*}{ Variáveis } & \multicolumn{2}{|c|}{ Empresas no Brasil } & \multicolumn{2}{c|}{ Empresas no Canadá } & \multicolumn{2}{c}{ Amostra total } \\
\cline { 2 - 7 } & Modelo I & Modelo II & Modelo I & Modelo II & Modelo I & Modelo II \\
\hline Intercepto & $0,2908^{* *}$ & $-0,3964$ & 0,6911 & 0,8886 & $-0,1232$ & 0,0333 \\
TAM & 0,003999 & $-0,0228$ & $0,0153^{* *}$ & 0,0056 & $0,0275^{* *}$ & 0,0172 \\
Endiv & $-0,2362^{* * *}$ & 0,0326 & $-0,0672$ & 0,1005 & 0,0424 & $-0,0218$ \\
Rent & $-0,1625$ & 0,1051 & $-0,0940$ & 0,111 & $-0,0843$ & $-0,0002$ \\
Merc & & $-0,0004$ & & 0,0001 & 0,0104 \\
Educ & & 0,0403 & & $-0,0638^{* *}$ & 0,0484 \\
Ccorrup & & $0,0582^{* * *}$ & & $-0,0892^{* * *}$ & $0,0322^{* *}$ \\
Trab & & $0,0804 * * *$ & & $0,1443 *$ & $-0,0001$ \\
\hline $\mathrm{R}^{2}$ & 0,1003 & 0,1584 & 0,0342 & 0,1227 & 0,0740 & 0,0549 \\
$\mathrm{R}^{2}$-ajustado & 0,0743 & 0,0995 & 0,0211 & 0,0664 & 0,0494 & 0,0244 \\
Teste F & $3,8638^{* *}$ & $2,6353^{* *}$ & $2,6090^{*}$ & $2,0816^{* *}$ & $3,0090^{* *}$ & $1,7952^{*}$ \\
Efeito do Painel & $\mathrm{P}$ & $\mathrm{A}$ & $\mathrm{P}$ & $\mathrm{A}$ & $\mathrm{P}$ & $\mathrm{A}$ \\
\hline
\end{tabular}

Fonte: Elaborada com base nos dados da pesquisa.

Níveis de significância: $*<0,1 ; * *<0,05 ; * * *<0,01$. Efeitos do painel: A - Aleatório; P - pooled ols.

Quando as variáveis financeiras são consideradas em conjunto com as variáveis do SNN, acabam perdendo sua significância. Observa-se significância estatística apenas para o controle de corrupção, a 1\% para Brasil e Canadá e com relações opostas, sendo observada uma influência positiva sobre as empresas brasileiras e negativa sobre as canadenses. Esta variável também é significativa para o modelo que engloba ambas as empresas, com nível de significância de $5 \%$ e efeito positivo sobre o DSC. Outras variáveis que demostraram significância estatística foram a relação entre sindicatos e empregadores para o Brasil, a 1\%, e para o Canadá, a $10 \%$, em ambos os casos exercendo influência positiva. Para o Canadá ainda se observou uma significância estatística de 5\% para a educação, com um efeito negativo sobre o desempenho social corporativo.

$\mathrm{Na}$ Tabela 10, para a dimensão social, quando são consideradas apenas as variáveis financeiras, o comportamento observado assemelha-se ao que se viu para o DSC, isto é, endividamento com significância negativa a $1 \%$ para o Brasil, e tamanho exercendo influência positiva sobre as empresas canadenses e na amostra completa, a $5 \%$ e $10 \%$, respectivamente. Diferente do que ocorreu no DSC, quando as variáveis financeiras são consideradas em conjunto com as do SNN, só perdem a significância para a amostra completa, enquanto para Brasil e Canadá, há uma troca do que é significante entre tamanho, que passa a ser significativamente negativo (a $10 \%$ ) sobre a DS das empresas brasileiras, e endividamento, que demonstra uma relação positiva e significante (a 5\%) para as empresas canadenses.

Entre as variáveis educação, controle de corrupção e relação entre sindicatos e empregadores se mostraram significantes para explicar a DS de ambos os países. No caso brasileiro, as duas primeiras variáveis apresentaram relação positiva com significância de $5 \%$, mesmo sinal da terceira variável, no entanto, com significância de $1 \%$. Para as empresas canadenses, a educação e o controle de corrupção apresentaram sinal negativo, com significância de $5 \%$ e $1 \%$, respectivamente, enquanto a relação entre sindicatos e empregadores demonstrou relação positiva com nível de significância de 5\%.

Tabela 10 - Regressão de dados em painel para a Dimensão Social (DS)

\begin{tabular}{c|cc|cc|cc}
\hline \multirow{2}{*}{ Variáveis } & \multicolumn{2}{|c|}{ Empresas no Brasil } & \multicolumn{2}{c|}{ Empresas no Canadá } & \multicolumn{2}{c}{ Amostra total } \\
\cline { 2 - 7 } & Modelo I & Modelo II & Modelo I & Modelo II & Modelo I & Modelo II \\
\hline Intercepto & $0,3587^{* *}$ & - & $-0,1326$ & - & 0,0889 & - \\
TAM & 0,0001 & $-0,0797^{*}$ & $0,0250^{* *}$ & $-0,0272$ & $0,0119^{*}$ & $-0,0123$ \\
Endiv & $-0,3006^{* * *}$ & 0,0772 & 0,0454 & $0,1526^{* *}$ & $-0,0804^{*}$ & 0,0785 \\
Rent & $-0,1384$ & 0,1076 & $-0,0974$ & 0,1083 & $-0,0651$ & 0,0217 \\
Merc & & $-0,0004$ & & $-0,0001$ & & $-0,0001$
\end{tabular}

Revista de Gestão Social e Ambiental - RGSA, São Paulo, v. 12, n. 3, p. 39-57, set./dez. 2018. 


\begin{tabular}{c|cc|cc|cc} 
Educ & & $0,0727^{* *}$ & & $-0,0688^{* *}$ & & 0,0273 \\
Ccorrup & & $0,0714^{* *}$ & & $-0,0884^{* * *}$ & & $-0,0063$ \\
Trab & & $0,1356^{* * *}$ & & $0,1885^{* *}$ & & $0,0547^{* *}$ \\
\hline $\mathrm{R}^{2}$ & 0,1003 & 0,2003 & 0,074 & 0,1634 & 0,0267 & 0,058 \\
$\mathrm{R}^{2}$-ajustado & 0,0743 & $-0,0066$ & 0,0494 & $-0,0549$ & 0,0135 & $-0,1467$ \\
Teste F & $3,8638^{* *}$ & $3,0420^{* * *}$ & $3,0080^{* *}$ & $2,5665^{* *}$ & 2,0230 & 1,6195 \\
Efeito do Painel & $\mathrm{P}$ & $\mathrm{F}$ & $\mathrm{P}$ & $\mathrm{F}$ & $\mathrm{P}$ & $\mathrm{F}$ \\
\hline
\end{tabular}

Fonte: Elaborada com base nos dados da pesquisa.

Níveis de significância: $*<0,1 ; * *<0,05 ; * * *<0,01$.

Efeitos do painel: A - Aleatório; F - Fixo; P - pooled ols.

Na Tabela 11, considerando a relação entre as variáveis de controle e a dimensão ambiental, nota-se que nenhuma delas foi significante para as empresas brasileiras, enquanto o tamanho demonstrou relação positiva e significante a $5 \%$ para as empresas canadenses. Para a amostra completa, observou-se o mesmo comportamento que o das empresas do Canadá. Para a dimensão ambiental, foi observado o mesmo comportamento que o DSC quando as variáveis são analisadas simultaneamente, ou seja, as variáveis financeiras perdem sua significância estatística.

Entre as variáveis do SNN, destaca-se, em ambos os países, o controle da corrupção, com efeito positivo no Brasil, e negativo quando se consideram as empresas canadenses e a amostra completa, com significância estatística de 5\% para o primeiro e último caso, e de $1 \%$ para o Canadá.

Tabela 11 - Regressão de dados em painel para a Dimensão Ambiental (DA)

\begin{tabular}{c|ccc|ccc}
\hline \multirow{2}{*}{ Variáveis } & \multicolumn{2}{|c|}{ Empresas no Brasil } & \multicolumn{2}{|c}{ Empresas no Canadá } & \multicolumn{2}{c}{ Amostra total } \\
\cline { 2 - 6 } & Modelo I & Modelo II & Modelo I & Modelo II & Modelo I & Modelo II \\
\hline Intercepto & $-0,0977$ & $-0,1668$ & $-0,1130$ & $1,1388^{*}$ & 0,0480 & 0,031 \\
TAM & 0,0257 & $-0,0166$ & $0,0303^{* *}$ & 0,0128 & $0,0189^{* *}$ & 0,0175 \\
Endiv & 0,0817 & 0,0611 & 0,0393 & 0,0798 & $-0,0532$ & 0,0442 \\
Rent & 0,0193 & 0,1097 & $-0,0703$ & 0,146 & $-0,1247$ & 0,044 \\
Merc & & $-0,0004$ & & 0,0001 & & $-0,0001$ \\
Educ & & 0,0176 & & $-0,0665^{*}$ & & $0,031^{* *}$ \\
Ccorrup & & $0,0693^{* *}$ & & $-0,1131^{* * *}$ & & $-0,0265^{* *}$ \\
Trab & & 0,0364 & & 0,1348 & & $-0,0342$ \\
\hline R & 0,0942 & 0,1906 & 0,0610 & 0,1234 & 0,0368 & 0,0829 \\
$\mathrm{R}^{2}$-ajustado & 0,0681 & 0,1339 & 0,0361 & 0,0671 & 0,0237 & 0,0533 \\
Teste F & $3,2006^{* *}$ & $3,3054^{* * *}$ & $2,4472^{*}$ & $2,0467 *$ & $2,8162^{* *}$ & $2,7987^{* * *}$ \\
Efeito do Painel & $\mathrm{A}$ & $\mathrm{A}$ & $\mathrm{P}$ & $\mathrm{A}$ & $\mathrm{A}$ & $\mathrm{A}$ \\
\hline
\end{tabular}

Fonte: Elaborada com base nos dados da pesquisa.

Níveis de significância: $*<0,1 ; * *<0,05 ; * * *<0,01$.

Efeitos do painel: A - Aleatório; $\mathrm{P}$ - pooled ols.

Cabe destacar que, apesar das variáveis independentes serem ligadas aos países, isto é, todas as empresas do mesmo país no mesmo ano receberem o mesmo valor, os modelos não apresentaram problemas de multicolinearidade, sendo o valor mais elevado do VIF observado de 4,2447, para a variável educação, quando são consideradas as variáveis de ambos os países para explicar a dimensão ambiental do DSC. Com base nos resultados obtidos, a Tabela 12 apresenta uma síntese dos resultados e sua relação com as hipóteses elencadas para o DSC.

Tabela 12 - Síntese dos resultados obtidos para o desempenho social corporativo

\begin{tabular}{|c|c|c|c|c|}
\hline Relacionamento & Hipótese & Brasil & Canadá & Ambos os Países \\
\hline $\mathrm{TAM} \rightarrow \mathrm{DSC}$ & $\mathrm{H}_{1}:$ Efeito Positivo & Rejeitada & Aceita & Aceita \\
\hline Rent $\rightarrow$ DSC & $\mathrm{H}_{2}:$ Efeito Positivo & Rejeitada & Rejeitada & Rejeitada \\
\hline Endv $\rightarrow$ DSC & $\mathrm{H}_{3}:$ Efeito Negativo & Aceita & Rejeitada & Rejeitada \\
\hline Educ $\rightarrow$ DSC & $\mathrm{H}_{4}$ Efeito Positivo & Rejeitada & Rejeitada & Rejeitada \\
\hline Ccorrup $\rightarrow$ DSC & $\mathrm{H}_{5}:$ Efeito Positivo & Aceita & Rejeitada & Aceita \\
\hline
\end{tabular}




\begin{tabular}{|c|c|c|c|c|}
\hline Merc $\rightarrow$ DSC & $\mathrm{H}_{6}:$ Efeito Positivo & Rejeitada & Rejeitada & Rejeitada \\
\hline Trab $\rightarrow$ DSC & $\mathrm{H}_{7}$ : Efeito Positivo & Aceita & Aceita & Rejeitada \\
\hline
\end{tabular}

Fonte: Elaborada pelos autores

De modo geral, no caso do Brasil, são aceitas a influência negativa do endividamento e a influência positiva dos sistemas político e trabalhista no DSC. No caso do Canadá, são aceitas as hipóteses da influência do tamanho da empresa e do sistema trabalhista no DSC. Quando os dois países são analisados em conjunto, observa-se que apenas o tamanho da empresa e o controle da corrupção influenciam positivamente o DSC.

\section{DISCUSSÃO}

A pesquisa confirma a influência de características financeiras e do sistema nacional de negócios no desempenho social corporativo, em países distantes institucionalmente, como o Brasil e Canadá. Os resultados parecem indicar, no Brasil, a predominância do isomorfismo coercitivo e normativo. No entanto, no caso do Canadá parece predominar o isomorfismo normativo e cognitivo. Os resultados da pesquisa indicam um maior poder discricionário, que se traduz em práticas mais oportunas, das empresas brasileiras, uma vez que o país tem um ambiente institucional muito mais permissivo do que o canadense.

Dessa forma, apesar do desempenho social corporativo das empresas brasileiras ser superior, as diferenças entre os dois países não são estatisticamente significantes. $\mathrm{O}$ ambiente institucional brasileiro permite às empresas uma adoção maior de práticas explícitas de RSC. Por outro lado, as empresas canadenses estão mais limitadas a práticas implícitas, uma vez que estão sujeitas a maiores pressões, quer seja por parte dos investidores no mercado financeiro, quer seja pela existência de uma forte e eficiente política de welfare state.

Ao analisar a diferença entre os indicadores do Brasil e no Canadá, percebe-se que a diferença entre os países se concentra principalmente nos indicadores sociais, em especial aqueles que se referem ao combate de trabalho infantil ou trabalho escravo. Rezende e Rezende (2013), e Ramalho e Mesquita (2013) classificam, respectivamente, o trabalho escravo e o infantil como graves problemas que ainda devem ser combatidos pela sociedade brasileira. Strauss e McGrath (2016) chamam a atenção para o problema do trabalho em condições precárias de imigrantes no Canadá, o qual é combatido pelo Temporary Foreign Worker Program (TFWP). Cabe ressaltar que "trabalho em condições precárias" não necessariamente envolvem trabalho infantil ou trabalho escravo. Assim, percebe-se que há no Brasil uma margem maior para que empresas adotem práticas de RSC explícitas, no que tange a questão do trabalho escravo, o que acaba elevando seu desempenho social corporativo, quando comparado com o Canadá.

Com relação as características financeiras, os resultados da pesquisa divergem dos achados de Orlitzky et al. (2015), que encontraram uma maior importância de características financeiras sobre a determinação do desempenho social corporativo. Cabe ressaltar, no entanto, que o estudo de Orlitzky et al. (2015) utilizou empresas de países desenvolvidos. Nesse sentido, Lourenço e Branco (2013) sugerem que as características financeiras são mais importantes na determinação do desempenho social corporativo de empresas em países emergentes, quando comparadas a empresas em países desenvolvidos. Os resultados da pesquisa reforçam a influência do tamanho para as empresas canadenses e o endividamento para as empresas brasileiras no DSC.

No caso dos SNN, os resultados evidenciam a importância do controle de corrupção sobre a propensão a práticas de RSC das empresas. Observou-se uma associação positiva e significante desta variável com o DSC das empresas brasileiras, e uma relação inversa e significante para as empresas canadenses, com uma ausência de significância para a amostra em geral. Luo (2006) corrobora com os resultados encontrados para o Brasil, na medida que evidencia que multinacionais, quando atuam em ambientes corruptos, têm maior propensão a adotar práticas 
antiéticas. Relação de mesma natureza foi encontrada por Ioannou e Serafeim (2012), que reportaram uma associação positiva entre a ausência de corrupção e o DSC.

No Brasil, há uma forte captação de recursos financeiros do poder público por parte do setor privado. A corrupção no Brasil e sua recorrência é fruto do legado cultural histórico brasileiro, apesar de outros fatores também contribuírem para a institucionalização da corrupção (Barquero, 2015). Para Taylor (2010), a corrupção no Brasil é um dos principais problemas do país por afetar seu desempenho econômico, além de reduzir a confiança nas instituições.

Klotz (2013), destaca o esforço por parte das empresas canadenses para criarem programas anticorrupção no país. Segundo Boisvert, Dent e Quraishi (2014), a forma de corrupção mais evidente no Canadá é aquela que envolve agentes públicos estrangeiros, o que não implica necessariamente, que o ambiente institucional canadense seja corrupto. De fato, Escresa e Picci (2015) descrevem que, entre 1998 e 2012, nenhum caso de corrupção de agentes públicos canadenses foi reportado. Klotz (2013) atribui à força da lei canadense contra esse tipo de prática.

Considerando a qualidade das relações entre sindicatos e empregadores, o resultado indica que à medida que os empregados aumentam seu poder coletivo são capazes de exigir melhores práticas sociais. Chih et al. (2010) também encontram uma relação positiva entre o DSC e a qualidade das relações entre empregado e empregadores. De modo semelhante, Ioannou e Serafeim (2012) encontram uma relação positiva e significante para a intensidade de atuação dos sindicatos sobre o DSC.

\section{CONSIDERAÇÕES FINAIS}

A utilização de indicadores para as características financeiras e as estruturas institucionais que moldam o SNN permitem novos insights sobre o desempenho social corporativo. No campo gerencial, os resultados indicam que fatores externos à organização são capazes de influenciar sua estratégia. No campo teórico, o trabalho agrega à discussão sobre a influência do ambiente institucional no DSC. Os resultados demonstram que o Canadá tem um conjunto claro de limites e parâmetros que orientam o ambiente institucional.

Em contraste, no Brasil, estas fronteiras não estão claramente definidas e permitem experimentação de novas abordagens de RSC (Abreu et al., 2015). Para o Brasil, uma lição aprendida nesta pesquisa, é a necessidade de um melhor controle da corrupção para ampliar o DSC. Paralelamente, o endividamento das empresas exerce uma forte influência negativa no DSC. E, um melhor relacionamento entre empregado e empregador pode ter efeitos positivos no DSC de empresas brasileiras.

Como limitações da pesquisa, aponta-se, o horizonte temporal adotado, que reduziu as alternativas de indicadores que poderiam ser utilizados para representar o ambiente institucional. Outra limitação consiste na mensuração do desempenho social corporativo. Apesar de ter sido utilizada uma metodologia proposta por Fisher e Sawczyn (2012), a evidenciação de práticas de RSC é apenas um dos aspectos do desempenho social corporativo.

Embora se reconheçam as limitações empíricas do trabalho, estudos comparativos em países institucionalmente distantes podem melhorar o entendimento da influência de pressões isomórficas coercitivas, normativas e cognitivas no desempenho social corporativo.

\section{REFERÊNCIAS}

Abreu, M. C. S., Cunha, L. T. \& Barlow, C. Y. (2015). Institutional dynamics and organizations affecting the adoption of sustainable development in the United Kingdom and Brazil. Business Ethics: A European Review, 24(1), pp. 73-90. 
Aguinis, H. (2011). Organizational responsibility: doing good and APA Handbooks in Psychology. APA handbook of industrial and organizational psychology, 3. Maintaining, expanding, and contracting the organization, 855-879. Washington, DC, US: American Psychological Association.

Aguinis, H., \& Glavas, A. (2012). What we know and don't know about corporate social responsibility: a review and research agenda. Journal of management, 38(4), 932-968.

Amable, B. (2003). The diversity of modern capitalism. Oxford University Press on Demand.

Artiach, T., Lee, D., Nelson, D. \& Walker, J. (2010). The determinants of corporate sustainability performance. Accounting \& Finance, 50(51).

Barquero, M. (2015). Corruption, political culture and negative social capital in Brazil. Revista Debates, 9(2), 139-157.

Boisvert, A., Dent, P. \& Quraishi, O. B. (2014) Corruption in Canada: definitions and enforcement. Public Safety Canada.

Brito, G. A., Corrar, L. J. \& Batistella, F. D. (2007). Fatores determinantes da estrutura de capital das maiores empresas que atuam no Brasil. Revista Contabilidade \& Finanças, 18(43), 9-19.

Cai, Y., Jo, H. \& Pan, C. (2012). Doing well while doing bad? CSR in controversial industry sectors. Journal of Business Ethics, 108(4), 467-480.

Simpson, W. G., \& Kohers, T. (2002). The link between corporate social and financial performance: evidence from the banking industry. Journal of business ethics, 35(2), 97-109.

Chih, H., Chih, H., \& Chen, T. (2010). On the determinants of Corporate Social Responsibility: international evidence on the financial industry. Journal of Business Ethics, 93, 115-135.

Croissant, Y. \& Millo, G. (2008). Panel data econometrics in R: the plm package. Journal of Statistical Software, 27(2).

Du, S., Bhattacharya, C. B., \& Sen, S. (2010). Maximizing business returns to corporate social responsibility (CSR): the role of CSR communication. International journal of management reviews, 12(1), 8-19.

Escresa, L., \& Picci, L. (2016). Trends in corruptions around the world. European Journal on Criminal Policy and Research, 22(3), 543-564.

Esteban-Sanchez, P., de la Cuesta-Gonzalez, M., \& Paredes-Gazquez, J. D. (2017). Corporate social performance and its relation with corporate financial performance: international evidence in the banking industry. Journal of cleaner production, 162, 1102-1110.

Fischer, T. M. \& Sawczyn, A. A. (2013). The relationship between corporate social performance and corporate financial performance and the role of innovation: evidence from German listed firms. Journal of Management Control, 24(1), pp. 27-52.

Freeman, R. E. (1984). Strategic management: a stakeholder perspective. Boston: Pitman, 13.

Friedman, M. (2000). Canada and flexible exchange rates. In speech at "Revisiting the Case for Flexible Exchange Rates." A conference sponsored by the Bank of Canada, Ottawa, Ontario, November.

Funderburk, C. (2012). Political corruption in comparative perspective: sources, status and prospects. Nova Iorque: Routledge.

Hasan, I., Kobeissi, N., Liu, L., \& Wang, H. (2018). Corporate social responsibility and firm financial performance: the mediating role of productivity. Journal of Business Ethics, 149(3), 671688. 
Heritage Foundation (2017). The index of economic freedom. Washington, D.C: Heritage Foundation.

Inoue, Y., \& Lee, S. (2011). Effects of different dimensions of corporate social responsibility on corporate financial performance in tourism-related industries. Tourism Management, 32(4), 790 804.

Ioannou, I. \& Serafeim, G. (2012). What drives corporate social performance? The role of nationlevel institutions. Journal of International Business Studies, 43(9), 834-864.

Jamali, D. \& Mirshark, R. (2007). Corporate social responsibility (CSR): theory and practice in a developing country context. Journal of Business Ethics, 72(3), 243-262.

Karam, C. M., \& Jamali, D. (2013). Gendering CSR in the Arab Middle East: an institutional perspective. Business Ethics Quarterly, 23(1), 31-68.

Kim, K. H., Kim, M., \& Qian, C. (2018). Effects of corporate social responsibility on corporate financial performance: a competitive-action perspective. Journal of Management, 44(3), 10971118.

Klotz, J. M. (2013). The anti-corruption dilemma for Canadian companies: just how far must companies go to comply with the law? Miller Thomson LLP.

Lourenço, I. C. \& Branco, M. C. (2013). Determinants of corporate sustainability performance in emerging markets: the Brazilian case. Journal of Cleaner Production, 57, 134-141.

Luo, T. (2006). Political behavior, social responsibility, and perceived corruption: A structuration perspective. Journal of International Business Studies, 37(6), 747-766.

Maignan, I., \& Ralston, D. A. (2002). Corporate social responsibility in Europe and the US: Insights from businesses' self-presentations. Journal of International Business Studies, 33(3), 497-514.

Margolis, J. D., \& Walsh, J. P. (2003). Misery loves companies: rethinking social initiatives by business. Administrative science quarterly, 48(2), 268-305.

Matten, D. \& Moon, J. (2008). "Implicit" and "explicit" CSR: a conceptual framework for a comparative understanding of corporate social responsibility. Academy of Management Review, $33(2), 404-424$.

Orlitzky, M., Schmidt, F. L., \& Rynes, S. L. (2003). Corporate social and financial performance: A meta-analysis. Organization studies, 24(3), 403-441.

Orlitzky, M.; Louche, C.; Gond, J. \& Chapple, W. (2015). Unpaking de drivers of corporate social performance: a multilevel, multistakeholder, and multimethod analysis. Journal of Business Ethics, $1-20$.

Ramalho, H. M. D. B., \& Mesquita, S. P. D. (2013). Determinantes do trabalho infantil no Brasil urbano: uma análise por dados em painel 2001-2009. Economia Aplicada, 17(2), 193-225.

Rezende, M. J., \& Rezende, R. C. (2013). As dificuldades de erradicação do trabalho escravo no Brasil hoje e a exposição dos muitos desafios postos ao desenvolvimento humano. Nómadas.

Roberts, R. W. (1992). Determinants of corporate social responsibility disclosure: an application of stakeholder theory. Accounting, Organizations and Society, 17(6), 595-612.

Ruf, B. M., Muralidhar, K., Brown, R. M., Janney, J. J. \& Paul, K. (2001). An empirical investigation of the relationship between change in corporate social performance and financial performance: a stakeholder theory perspective. Journal of Business Ethics, 32(2), pp. 143-156.

Schrammel, T. (2013) Bridging the institutional void: an analytical concept to develop valuable cluster services. Management Revue, 24 (2), 114-132. 
Scott, W. R. (1995). Institutions and organizations. Foundations for organizational science. London: A Sage Publication Series.

Surroca, J., \& Tribó, J. A. (2008). Managerial entrenchment and corporate social performance. Journal of Business Finance \& Accounting, 35(5-6), 748-789.

Thorne, L., Mahoney, L. S., Gregory, K., \& Convery, S. (2017). A comparison of Canadian and US CSR strategic alliances, CSR reporting, and CSR performance: Insights into implicit-explicit CSR. Journal of Business Ethics, 143(1), 85-98.

Ullmann, A. A. (1985). Data in search of a theory: A critical examination of the relationships among social performance, social disclosure, and economic performance of US firms. Academy of Management Review, 10(3), 540-557.

UNPD (2016) Human Development Report 2016: Human Development for Everyone. Nova Iorque: United Nations Development Programme.

Whitley, R. (1999). Divergent capitalisms: the social structuring and change of business systems. Oxford: Oxford University Press.

Wood, D. J. (1991). Corporate social performance revisited. Academy of management review, 16(4), 691-718.

Data da submissão: 20/12/2017

Data de aceite: 13/08/2018 